\title{
Tactical Conflict Detection in Terminal Airspace
}

\author{
Huabin Tang ${ }^{1}$ and John Robinson ${ }^{2}$ \\ NASA Ames Research Center, Moffett Field, CA 94035 \\ and \\ Dallas Denery ${ }^{3}$ \\ University of California, Santa Cruz
}

\begin{abstract}
Air traffic systems have long relied on automated short-term conflict prediction algorithms to warn controllers of impending conflicts (losses of separation). The complexity of terminal airspace has proven difficult for such systems as it often leads to excessive false alerts. Thus, the legacy system, called Conflict Alert, which provides short-term alerts in both en-route and terminal airspace currently, is often inhibited or degraded in areas where frequent false alerts occur, even though the alerts are provided only when an aircraft is in dangerous proximity of other aircraft. This research investigates how a minimal level of flight intent information may be used to improve short-term conflict detection in terminal airspace such that it can be used by the controller to maintain legal aircraft separation. The flight intent information includes a site-specific nominal arrival route and inferred altitude clearances in addition to the flight plan that includes the RNAV (Area Navigation) departure route. A new tactical conflict detection algorithm is proposed, which uses a single analytic trajectory, determined by the flight intent and the current state information of the aircraft, and includes a complex set of current, dynamic separation standards for terminal airspace to define losses of separation. The new algorithm is compared with an algorithm that imitates a known en-route algorithm and another that imitates Conflict Alert by analysis of false-alert rate and alert lead time with recent real-world data of arrival and departure operations and a large set of operational error cases from Dallas/Fort Worth TRACON (Terminal Radar Approach Control). The new algorithm yielded a false-alert rate of two per hour and an average alert lead time of 38 seconds.
\end{abstract}

\section{Introduction}

$\mathrm{T}$ HE current U.S. air transportation system is expected to be unable to support the projected demand for air travel. One of the primary limitations is controller workload. A combination of air- and ground-based automation is a potential solution to the limitation of airspace capacity due to controller workload. One area of research central to this goal is the automation of conflict detection and resolution functions. ${ }^{1}$

Recently there has been considerable research directed towards a new concept for automated separation assurance, referred to as the Advanced Airspace Concept (AAC). ${ }^{2-4}$ The AAC provides two independent layers of separation assurance - a strategic layer and a tactical layer. The strategic layer focuses on mid-term conflicts (losses of separation) predicted to be from 2 to 20 minutes into the future. The tactical layer addresses short-term or imminent conflicts predicted to occur within approximately 2 minutes. A third layer of safety is provided by an independent airborne collision avoidance system such as TCAS (Traffic Alert and Collision Avoidance System). ${ }^{5}$ TCAS deals with potential collisions less than approximately 45 seconds away. The tactical layer, known as Tactical Separation Assured Flight Environment (TSAFE), is proposed as a backup system that duplicates a limited set of safety-critical functions of the strategic layer. TSAFE simplifies the problem of automated separation assurance and provides a safety net for the strategic layer. Most of the research to date has focused on en-route airspace. En-route prototypes of TSAFE have been developed and studied with the use of en-route operational error cases ${ }^{6-9}$. Some

\footnotetext{
${ }_{1}^{1}$ Aerospace Engineer, AFA 210-6, Moffett Field, CA 94035, huabin.tang-1@ nasa.gov.

2 Aerospace Engineer, AFC 210-10, Moffett Field, CA 94035, john.e.robinson@ nasa.gov, Member AIAA.

${ }^{3}$ Senior Scientist, University of California, Santa Cruz, ddenery@ucsc.edu, Fellow AIAA.
} 
attention on TSAFE conflict resolution has also appeared recently. ${ }^{10-12}$ TSAFE as an independent system to aid the controller for the near term has also been proposed ${ }^{8-11}$ A high-level specification for Short Term Conflict Alert systems similar to TSAFE has also recently been released by EUROCONTROL. ${ }^{13}$

The complexity of terminal airspace has proven difficult for tactical conflict detection systems. The contributing factors include the dense air traffic, frequent large turns, an incompletely specified flight plan, a complex set of separation standards, and the frequent necessity to operate aircraft purposely near the required separation standards. Conflict Alert, ${ }^{14}$ the legacy system that relies mainly on dead reckoning to predict aircraft trajectories, currently provides tactical alerts in terminal airspace. It is designed to determine if two aircraft are in dangerous proximity of each other rather than if they are going to lose legal separation by the separation standards. It is often inhibited or degraded in areas where frequent false alerts occur. ${ }^{15}$ Thus, Conflict Alert was not designed for the controller to maintain legal aircraft separation, and as such the separation standards for terminal airspace have not been adapted closely enough. On the other hand, the conflict detection algorithms in en-route TSAFE have not been designed to address the unique problems of terminal airspace. To the best of our knowledge, no report on direct application of the en-route algorithms to terminal airspace can be found in the literature, and there is no literature on tactical conflict detection algorithm that covers the whole terminal airspace and follows the separation standards.

In this paper, a new conflict detection algorithm is proposed which uses a single analytic trajectory that takes into account available flight intent information and the current state of the aircraft. The trajectory consists of segments of straight lines and circular arcs that can be represented analytically. Apart from the flight plan, which can include a RNAV (Area Navigation) departure route, the flight intent information includes segments of nominal TRACON (Terminal Radar Approach Control) routes, speed restrictions, and altitude clearances inferred from the recorded track data. A complex set of current, dynamic separation standards for terminal airspace, as documented in the Federal Aviation Administration (FAA) Order JO 7110.65S,${ }^{16}$ is adapted to define losses of separation. The new algorithm is compared with an algorithm that imitates the dual-trajectory algorithm of en-route $\operatorname{TSAFE}^{9}$ and another that imitates Conflict Alert. The comparison is through the analysis of false-alert rate and alert lead time with fasttime simulation of recent real-world data of arrival and departure operations and 70 operational error cases from Dallas/Fort Worth TRACON. The results show that the new flight-intent algorithm yields a significantly reduced false-alert rate without much penalty in the alert lead time.

The rest of this paper is organized as follows. Sec. II presents a broad set of the standard FAA separation criteria used to define conflicts. Details about the tactical conflict detection algorithms are also presented. Sec. III provides results and discussion from analysis of the alerting performance of the algorithms. A classification of false alerts in the analysis of real-world traffic data is discussed as well. Sec. IV presents conclusions.

\section{Conflict Detection}

An operational error usually refers to the failure of an air traffic controller to detect a conflict with sufficient lead time to resolve it before loss of separation occurs. A key technical means of reducing operational errors in the near term would be to provide timely alerts of impending conflicts to controllers. This paper will not address the graphical user interface to alert controllers but instead focuses on the detection problem.

Conflict detection starts with predicting the trajectories of the flights involved. This relies on the aircraft's current state information and knowledge of the intended route. The vertical and horizontal separations are calculated along the predicted trajectories starting with the current positions. A loss of separation is found when the predicted separations are less than the standard separation criteria within the look-ahead period. No separation buffers are used in this research since any such buffer is unnecessarily subjective. The standard separation criteria in terminal airspace are dynamic, as they depend on the specific encounter geometry and the types of aircraft involved.

\section{A. Separation Criteria}

FAA Order JO $7110.65 \mathrm{~S}$ documents the required separation between aircraft. ${ }^{16}$ In the following subsections, the precise separation criteria used in this research are listed along with the assumptions made.

\section{General Separation Minima}

Aircraft in terminal airspace are generally required to maintain a separation of at least $3 \mathrm{nmi}$ horizontally or 1000 $\mathrm{ft}$ vertically. When a pair of aircraft is in transition from terminal to en route airspace, en route separation minima of $5 \mathrm{nmi}$ and $1000 \mathrm{ft}$ apply. An example as to when this criterion would be applied is when one of the aircraft is above the TRACON ceiling, which for DFW TRACON is at 17,000 ft. 


\section{Wake Separation Minima}

When a trailing aircraft operates horizontally within $2500 \mathrm{ft}$ of the flight path of the leading aircraft over the surface of the Earth, the trailing aircraft is said to be directly behind the leading aircraft. The wake turbulence separation minima in Table 1 are required when an aircraft

1) Operates directly behind and is either at the same altitude as, or within $1000 \mathrm{ft}$ below, another aircraft.

2) Follows another aircraft conducting an Instrument Landing System (ILS) approach.

Since it is impossible to tell based on the track data whether the aircraft are conducting ILS approaches, all approaches are treated as ILS approaches in this research. In addition, the aircraft must be Table 1 Wake separation for different weight classes in terminal airspace ensured to be able to maintain the separation minima in Table 2 when the leading aircraft is over the runway threshold.

When certain required conditions are satisfied, a reduced separation of $2.5 \mathrm{nmi}$ is authorized. These required conditions are as follows: (a) the leading aircraft's weight class is the same as or less than the trailing aircraft; (b) both aircraft are established on the final approach course within $10 \mathrm{nmi}$ of the

Table 2 Wake separation for different weight classes at runway threshold

\begin{tabular}{lccc}
\hline \hline Leading Aircraft & Heavy & B757 & Heavy \\
\hline Trailing Aircraft & Heavy & Small & Small \\
\hline Sep. Minima, nmi & 4 & 5 & 6
\end{tabular}

runway threshold; (c) an average runway occupancy time of 50 seconds or less was documented; (d) Certified Tower Radar Displays (CTRDs) were operational and used for quick glance references by controllers. In this research, we assume that conditions (c) and (d) are satisfied whenever conditions (a) and (b) are satisfied. Heavy and B757 (Boeing 757) aircraft are permitted to participate in this separation reduction as the trailing aircraft only. An aircraft is considered to be established on the final approach course (localizer) if it is within a given angular course width, measured from the localizer transmitter, which is typically $1000 \mathrm{ft}$ away from the far end of the runway. The course width of a final approach course is tailored to provide $700 \mathrm{ft}$ full scale at the threshold. ${ }^{17}$ A typical runway length of $9000 \mathrm{ft}$ is assumed in this research, which corresponds to a course width of about 4 degrees. In a real system, the localizer dimensions for a given runway are available and can be input to the system.

3. Other Separation Minima

1) When two aircraft are on parallel dependent ILS approaches to runways with a center-line separation of at least $2500 \mathrm{ft}$ but no more than $4300 \mathrm{ft}$, a minimum of $1.5 \mathrm{nmi}$ is required.

2) In the case of an arrival trailing a departure, a minimum of $2 \mathrm{nmi}$ and $1000 \mathrm{ft}$ must be maintained between the aircraft if the separation will increase to a minimum of $3 \mathrm{nmi}$ within 1 minute after the takeoff.

3) Between a VFR (Visual Flight Rules) aircraft and an IFR (Instrument Flight Rules) aircraft, the separation minima are $1.5 \mathrm{nmi}$ and $500 \mathrm{ft}$.

\section{Exceptions}

There are exceptions to the above minimum separation requirements when diverging courses are involved. Two aircraft are said to be on SAME, CROSSING, or OPPOSITE courses if the angular difference between their courses is contained in the mathematical intervals $\left[0,45^{\circ}\right),\left[45^{\circ}, 135^{\circ}\right]$, or $\left(135^{\circ}, 180^{\circ}\right]$ respectively. ${ }^{16}$ There is no loss of separation if two aircraft are

1) On SAME or CROSSING courses with their courses diverging by more than $15^{\circ}$ and one aircraft has crossed the projected course of the other.

2) On OPPOSITE courses and they have passed each other.

3) Successive departing aircraft separated by more than $1 \mathrm{nmi}$.

For successive departing aircraft, the standard criterion requires the courses to diverge by $15^{\circ}$ or more. However, many successive departures appear not to follow this divergence requirement and further investigation is needed to clarify this. Thus, the diverging requirement is relaxed in this paper.

Another exception case is when both aircraft are established on their independent final approach courses. In this case, there is no separation requirement. It is generally impossible to tell whether the runways are dependent or independent based on current track data. However, when their centerlines are separated by more than 4300 feet, they are used more often as independent runways. Thus, unless usage of dependent runways is stated explicitly, as in the operational error report, we assume that the runways are independent when their centerlines are separated by more 
than $4300 \mathrm{ft}$. In a real system, this can be input information. When the centerlines are separated by a distance within $2500 \mathrm{ft}$ and $4300 \mathrm{ft}$, dependent runways are expected and the 1.5 -nmi criterion as described above is used.

\section{B. Flight-Intent Routes and Horizontal Conformance}

Horizontal conformance is defined in terms of an aircraft's position relative to its flight intent route, which refers to the merging of its FAA flight-plan route and the nominal TRACON route segments. The FAA flight plan today generally does not have complete details from the meter fix to the runways. On the departure side, the RNAV departure route of the flight plan provides accurate enough waypoints from the departure runway to the meter fix. Nevertheless, the RNAV routes in the DFW TRACON are not available for every airport.

Aircraft generally follow the same nominal paths that have some common flexibility in the TRACON. Past air traffic automation efforts have used these prescribed nominal TRACON paths from the meter fixes down to the runways. ${ }^{18}$ They are sometimes referred to as nominal interior routes (NIRs). An NIR for an aircraft is unique given the airspace configuration and the engine type, meter fix, airport, and assigned runway. A typical NIR is shown in Fig. 1 where the squares on the center line indicate waypoints. The last two waypoints in the final leg are the final approach fix (FAF) and runway threshold fix (RWY). Most arrival aircraft in DFW TRACON are observed to follow the NIRs except that a base extension or "trombone" is common. Thus, an NIR approximately describes the horizontal flight intent of an arrival aircraft in today's system. The flight-intent route of an aircraft merges its flight-plan route with its NIR.

In this research, an aircraft is said to be in conformance or on-track if its cross-track distance to the center of the nominal interior route is within a conformance threshold; otherwise the aircraft is said to be out of conformance or off-track. Thus, as shown in

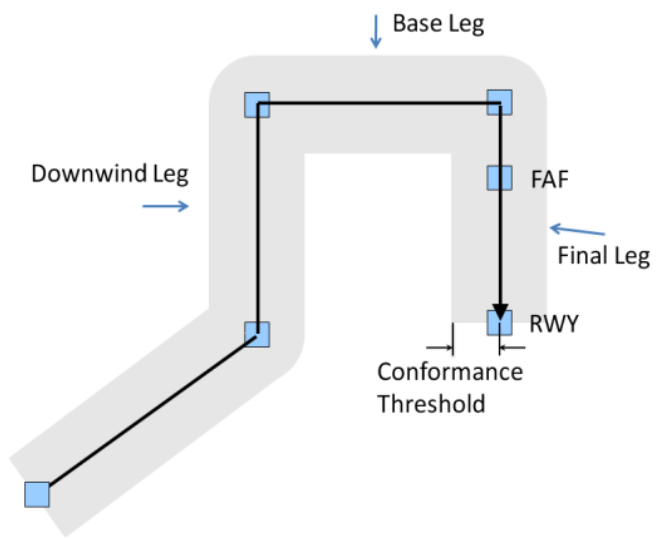

Figure 1. A static nominal interior route with a conformance region as indicated

Fig. 1, if an aircraft is within the shaded area defined by the conformance threshold, the aircraft is on-track. The value for the conformance threshold is taken to be $0.5 \mathrm{nmi}$ throughout. Conformance is used in constructing the horizontal track of an aircraft and the conformance threshold is based on qualitative comparison of a large number of predicted and actual horizontal tracks. For simplicity, aircraft heading is not considered in defining the conformance. For the purpose of constructing horizontal tracks, heading becomes less important in the conformance definition in terminal airspace because of the shorter radar update cycle of 4.8 seconds as compared to 12 seconds in the en route. The conformance threshold for final approach courses should be adjusted to optimize the detection of blunders in the final approach.

\section{Flight-Intent Trajectory}

A flight-intent (FI) trajectory algorithm is described which allows an aircraft trajectory to be constructed using segments of straight lines and circular arcs that can be represented analytically. Use is made of the flight-intent route and the current state information of the aircraft. A flyable horizontal track is first generated based on conformance and the flight-intent route. A ground speed profile is then created for the aircraft to fly along the track. Finally, an altitude profile is generated and superimposed on the horizontal trajectory. Additional flight intent data in the form of speed restrictions and altitude clearances may also be used.

\section{Horizontal Track}

The horizontal track for an FI trajectory assumes that, whenever possible, an aircraft attempts to conform to its flight intent route and other available intent information. Thus, when in conformance, it will stay in conformance; otherwise it will move along a straight line along its current course. However, the aircraft is assumed to be aware of its flight intent in the sense that, when possible, it joins smoothly back with the next segment in its FI route. So the aircraft flies along a horizontal track constructed using straight lines and circular arcs, which can be represented analytically, based on the following general rules:

1) If the aircraft is on-track, capture the next waypoint in the flight intent route.

2) If the aircraft is off-track, start with a straight line along its current course then, if possible, join the flightintent route when it is intercepted; otherwise continue along the straight line. 
The radius of the circular-arc segments, assuming a coordinated turn, is estimated from the aircraft's current ground speed, $V$, and a bank angle, $\phi_{B}$, by $r_{c}=V^{2} / g \tan \phi_{B}$, where $g$ is the acceleration of gravity and $\phi_{B}=30^{\circ}$. Since base extensions are common and turns onto the final are constrained, special rules apply to downwind-to-base and base-to-final turns. The rules are summarized as follows, with the numerical values being based on engineering experience: rules:

1) Downwind-to-Base Rule: First, before a turn is detected, the aircraft is predicted to continue along its velocity vector (DR). Downwind-to-Base Rule: First, before a turn is detected, the aircraft is predicted to continue along its velocity vector. That is, a downwind-to-base turn will not commence until an actual turn of the aircraft has been detected. This rule is based on the observation that the base leg of the NIR is extended in most cases. The detection of a turn is defined as three consecutive course changes in the same direction (left or right). After a turn towards the base is detected, the actual turn radius is calculated based on the current rate of course change, $\omega$, and the current ground speed by $r_{a}=V / \omega$. If the current heading of the aircraft is more than 150 degrees from the final approach course, the aircraft is predicted to continue turning for 10 seconds or about two radar update cycles with the current actual turn radius, $r_{a}$, and then continue along a straight-line projection at the end of the turn. If the current course of the aircraft is within 150 degrees of the final approach course, the aircraft is assumed to continue turning with radius, $r_{a}$, to a course perpendicular to the final approach course. If the turn is not possible because $r_{a}$ is too large, the coordinated turn radius, $r_{c}$, is tried. If the turn is still not possible, a straight line is used.

2) Base-to-Final Rule: An aircraft approaching the final approach course with some angle is generally assumed to turn and start to intercept the final approach course at some minimum perpendicular distance, $d$, see Fig.2 for a typical turn scenario. Based on visual inspection of many actual trajectories, we take $d=2 \mathrm{nmi}$. Circular arcs and straight lines are used to construct

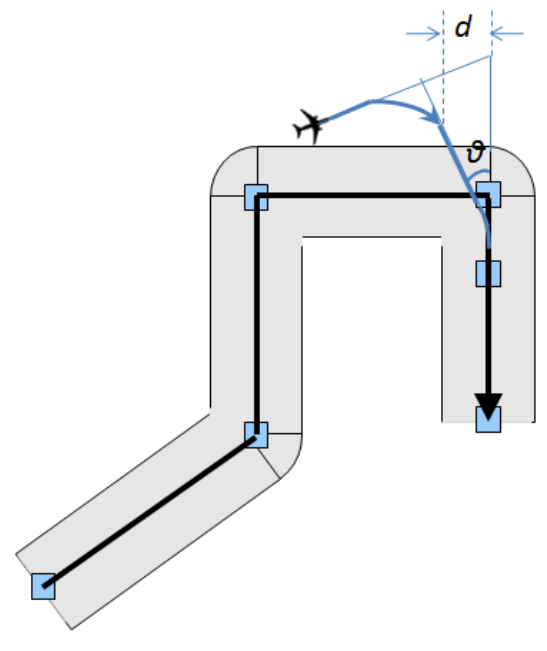

Figure 2. A typical interception of the final approach course

the trajectories of interception. An interception angle, $\theta$, of approximately $30^{\circ}$ before the final approach fix is assumed. If an aircraft is already closer than $2 \mathrm{nmi}$ to the final approach course, it is assumed to turn and intercept right away. If it is not possible to turn, it is assumed to fly along a straight line.

As an illustration, Fig. 3 shows the FI trajectories predicted at each radar track position for an aircraft following a nominal interior route. The dashed line is the static NIR, which connects the waypoints represented by the squares. The final approach fix (FAF) and runway threshold (RWY) are also indicated. The base was extended about $9.5 \mathrm{nmi}$. The circles and diamonds are the actual radar track positions with the circles indicating on-track and the diamonds off-track. The solid lines are the FI predictions. The intersection between the downwind and the expected base legs are indicated by label $\mathrm{A}$. The actual base and final turns are indicated by $\mathrm{B}$ and $\mathrm{C}$, respectively. Notice that the FI trajectories turn only after proper turns have been detected at $B$ instead of following the base turn of the NIR at A. Before the base turn at
Ground Tracks with FP Predictions

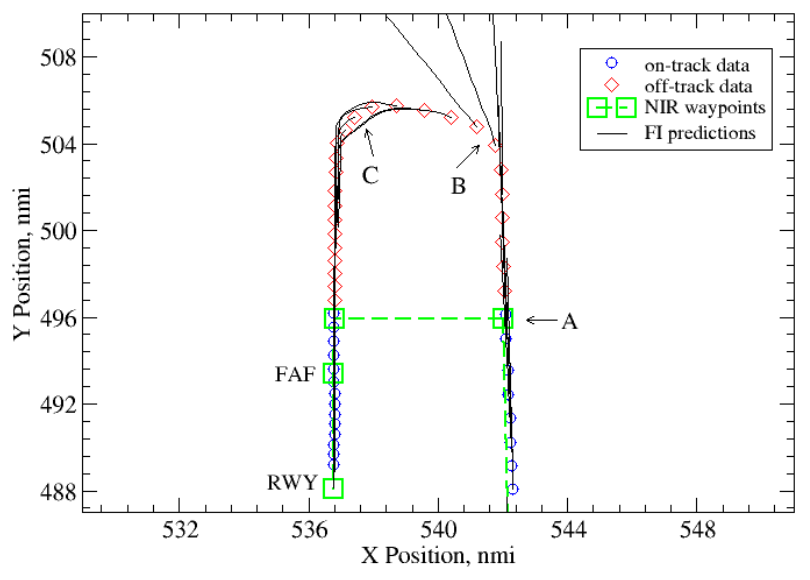

Figure 3. Sample ground track with predicted FI trajectories for an aircraft following a nominal interior route. 
B is detected, the aircraft follows the downwind leg and continues along its course in a straight line since it is offtrack. Then, when the turn at B is detected while the course of the aircraft is still more than $150^{\circ}$ from its final approach course, the aircraft turns 10 more seconds before continuing along a straight line. Once its course is less than $150^{\circ}$ from the final approach course, the aircraft turns into the base, approaches and intercepts properly the final approach course. For comparison, Fig.4 shows the corresponding dead-reckoning (DR) predictions (straight lines along the aircraft courses) for the same aircraft. As can be seen, the FI trajectories provide more accurate predictions than the DR trajectories.

2. Ground Speed Profile

A ground speed profile based on current ground speed and acceleration is generated for the aircraft to fly along the constructed horizontal track. Our experience from comparing the trajectory predictions with the actual TRACON tracks suggests that the ground speed changes significantly enough that it is necessary to model the acceleration. However, since the actual duration of deceleration of an aircraft is not known, the rate of the

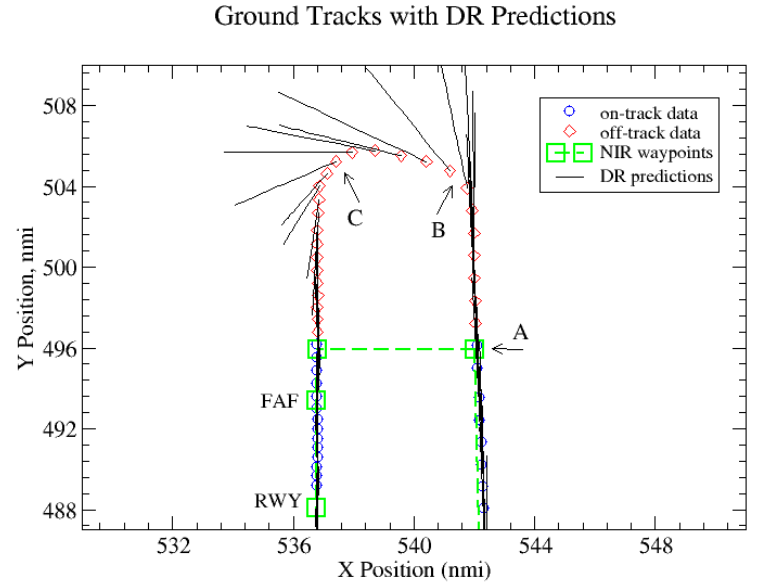

Figure 4. Sample ground track with predicted DR trajectories for an aircraft following a nominal interior route.

deceleration may be so large that the aircraft may be predicted to reduce its speed unrealistically to zero within the 2-minute probe period. Thus, a lower limit for the ground speed is necessary, especially during base leg and final approach periods. Similarly, the acceleration of a departing flight may be so large that an upper bound on the ground speed may need to be imposed. In this paper, ground speed lower bounds near the final approach and runway threshold fixes are imposed, and an upper bound is imposed on certain departure flights. Other bounds may be added in the future. When a ground speed bound is imposed, the aircraft is projected to fly at that speed bound once it is reached.

The lower bound on the ground speed near the final approach fix is set at 160 knots. The bound at the runway threshold is 130,115, or 95 knots depending on whether the engine type of the aircraft is jet, turboprop, or piston, respectively. The ground speed upper bound for departure flights is 260 knots for flights below $6500 \mathrm{ft}$. These numbers are based on observations of a large set of flight data. The results are not sensitive to the precise values. Given the current ground speed, the length of the constructed trajectory, and the speed bound, a required acceleration may be calculated. In the case of a deceleration, if the magnitude of the required deceleration is larger than the current measured value, the required value will be used. Otherwise, current ground deceleration is used. Note that while wind effects are not explicitly taken into account, they are implicitly taken into account through modeling the ground acceleration.

\section{Altitude Profile}

The climb or descent of an aircraft is simply modeled in a three-phase altitude profile: an initial acceleration phase, a constant-rate phase, and a final deceleration phase. A vertical constant acceleration of is assumed for the initial and final phases. With this acceleration, it takes about 10 seconds for an aircraft to increase its climb rate by $2000 \mathrm{fpm}$. Fig. 5 illustrates the model of a three-phase climb. The following rules are used to determine the phase of a flight:

1) When the vertical distance to the cleared altitudes of an aircraft is more than 200ft, and its climb or decent rate is more than $500 \mathrm{fpm}$, it is in the constant-rate phase.

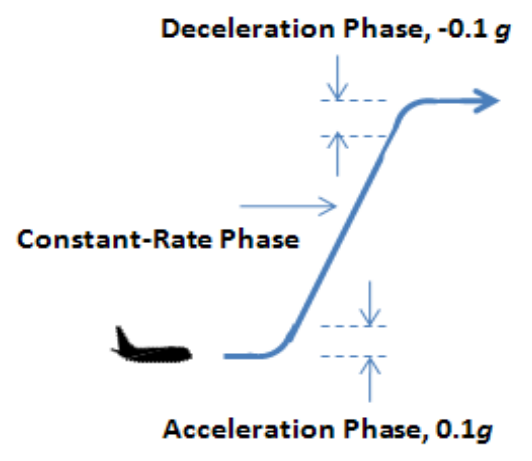

2) When the distance is more than $200 \mathrm{ft}$ and the climb or decent rate is less than $500 \mathrm{fpm}$, it is in the acceleration phase. (It is not in a deceleration phase since the speed would have to be larger for a stopping Figure 5. A typical interception of the final approach course distance of $200 \mathrm{ft}$.) 
3) Otherwise the aircraft is in the deceleration phase.

Note that the numbers above are adjustable and they appear reasonable when the predicted trajectory profiles are compared with many actual traffic trajectories. The conflict prediction results are not sensitive to the precise values. If an aircraft is in the constant-rate phase, its constant vertical rate is given by its current vertical rate. If an aircraft is in the initial acceleration phase, its vertical speed at the constant-rate phase is obtained by looking up the nominal climb or descent rate in the Base of Aircraft Data (BADA) from Eurocontrol. ${ }^{19}$

While most altitude clearances are entered into the Host computer at each Air Route Traffic Control Center (ARTCC or Center), ${ }^{20}$ these clearances usually are only transmitted by voice in the TRACON without being entered into the TRACON's ARTS or STARS computer. In the future, such cleared altitudes may be entered into the system, in which case the clearances would become available intent data for use in predicting the trajectory. To study the effects of temporary altitude clearances, we extract the location and duration of level segments from a recorded file of

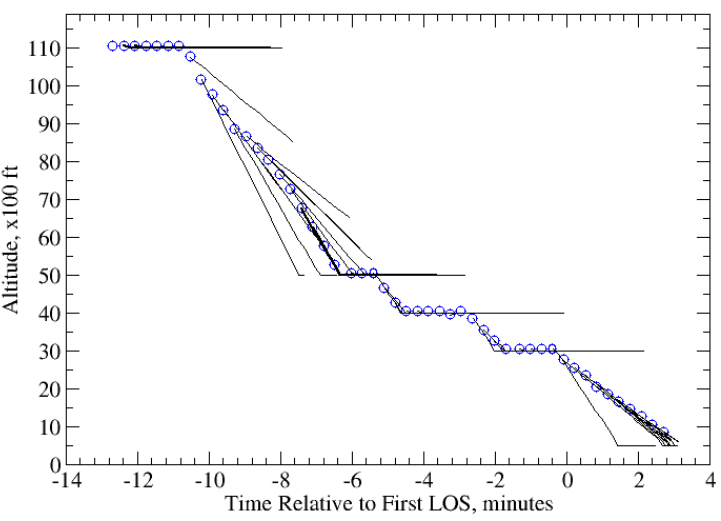

\section{Figure 6. Sample altitude profiles with inferred altitude} clearances. aircraft tracks and use them to generate simulated altitude clearances. The resulting cleared altitudes are referred to as Inferred Altitude Clearances (IACs) in this paper. By comparing the false-alert rates with and without the use of IACs, the importance of making them available can be addressed.

A sample altitude profile that uses IACs is shown in Fig. 6, where the circles represent the actual altitude above mean sea level (MSL) and the solid lines are predictions from the altitude profile algorithm. As seen in the figure, the predicted trajectories level off at the inferred altitudes. Without the IACs, the predicted trajectories would have continued descending below the cleared altitudes. Inevitably, this could cause false alerts.

\section{Conflict Prediction Algorithms}

An algorithm using the flight-intent trajectory is proposed to predict potential conflicts of aircraft in the terminal airspace. The algorithm, referred to as flight-intent (FI) conflict prediction algorithm, starts with the calculation of the flight-intent trajectories of all aircraft. Each trajectory consists of straight lines, circular arcs, kinematic groundspeed profile, and altitude profiles that can all be represented analytically. The positions of each pair of aircraft are then predicted and checked for any potential conflicts through the trajectories with an adjustable look-ahead period. When the horizontal track follows the flight-intent route, a look-ahead period of 2 minutes is used; otherwise a 1.5 minute look-ahead period is used instead.

As in en-route TSAFE and Conflict Alert, some heuristic methods are used to reduce false alerts caused by outlying track data as described below.

1) Alert Filtering: A 3-out-of-3 rule is applied if the predicted time to loss of separation (LOS) is larger than 60 seconds. That is, a loss of separation must be predicted for three consecutive radar updates. Similarly, a 2-out-of- 2 rule is applied if the predicted time to LOS is between 30 and 60 seconds. A 1-out-of- 1 rule (no repeat required) is applied if the predicted time to LOS is less than 30 seconds or if there exists an LOS prediction (not filtered by this rule) within the previous 25 seconds. The numbers used here are to suppress nuisance alerts due to outlying track positions, ground speeds, or vertical rates with proper consideration of the fact that current radar track update is 4.8 seconds. When the prediction time is below 30 seconds, it appears that the risk of delaying a critical alert due to filtering outweighs the benefit. The values should be made adjustable and further tuned in a real system.

2) Altitude Rounding: The standard altitude rounding rule that is used in the Host computer at each Center is adopted here. The rule is that any aircraft flying nominally level within $200 \mathrm{ft}$ of its cleared altitude is considered to be exactly at its cleared altitude for the purposes of separation requirements.

3) Initial Track Filtering: For the first or second radar updates, the course is usually not accurate, so those data points are excluded from conflict detection.

In general, the predicted states of a pair of aircraft at some look-ahead time determine whether a loss of separation shall be predicted. The situation is more complicated when wake turbulence is involved, since the track 
history is required to determine if the trailing aircraft is operating directly behind (within $2500 \mathrm{ft}$ of the flight path of) the leading aircraft. Thus, a 3-minute track history of each aircraft is kept. To check if wake separation applies to a pair of in-trail aircraft, the cross-track position of the trailing aircraft with respect to the track history of the leading aircraft must first be located. Then, if the altitude of the trailing aircraft is within $1000 \mathrm{ft}$ below that of the leading aircraft at the perpendicular cross-track position, wake separation applies.

To see how well the FI algorithm performs, two other prediction algorithms are designed. One algorithm, called the dead-reckoning (DR) algorithm, imitates that of Conflict Alert and uses DR trajectories obtained from straight lines along the aircraft courses and current ground speeds and accelerations of the aircraft. Another algorithm, called the dual-trajectory algorithm, imitates that of en-route TSAFE and uses both the DR and FI trajectories. These two algorithms are summarized below.

1) Dead-Reckoning Algorithm: DR trajectories are used to predict aircraft positions with a look-ahead period of 1.5 minutes. This reduced look-ahead period is chosen to reduce false alerts. When an aircraft is on its final approach course, determination of the separation criteria as described in Sec. II.A requires knowledge of the nominal interior route. Therefore, the nominal interior route is assumed to be known to the DR algorithm to determine separation criteria when the aircraft is on final approach. Otherwise, the DR algorithm would yield significantly more false alerts.

2) Dual-Trajectory Algorithm: Both DR and FI trajectories are used with 1.5- and 2-minute look-ahead periods, respectively. Furthermore, a vertical uncertainty envelope is introduced which allows vertical rate to vary within $\pm 10 \%$ of the constant rate in the constant-rate phase. Thus, a potential conflict is predicted when any of the four trajectory combinations, DR/DR, DR/FI. FI/DR, and FI/FI, between a pair of aircraft predicts one.

\section{Alerting Performance}

A prototype Terminal TSAFE system, built upon the test bed system in Ref. 12, has been developed that sets up a framework to incorporate different conflict detection and resolution algorithms in a fast-time simulation of aircraft traffic data. The input to the system is an archived data file containing radar tracking data, Mode $\mathrm{C}$ barometric altitude data, flight-plan route data, and altitude amendments. These input data are used to generate trajectories that are passed through the conflict detection algorithms to detect potential conflicts. Aircraft trajectories and conflict information are then recorded.

The FI conflict detection algorithm along with the DR and dual algorithms discussed in Sec. II.D were implemented in the prototype Terminal TSAFE system to evaluate and compare their performance in alerting the controller of potential conflicts. The results are discussed in the next two subsections.

\section{A. Alert Lead Times}

Alert lead time for predicting a loss of separation (LOS) is defined as the difference between the actual LOS time and the time of the first prediction. Thus, the larger the alert lead time the larger the predictive power of the system.

1. Variety of Operational Errors

The study of alert lead times is based on fast-time simulation of track data of 70 operational error cases from DFW TRACON during the period between January 2007 and April 2009. For each operational error case, a set of conflict plots for each detection algorithm was generated and examined along with the corresponding operational error report from the FAA. The operational errors covered a wide variety of situations as can be seen in Table 3. At least one aircraft was on final approach in 44 of the 70 cases. Fifteen cases involved aircraft coming from or going to different airports. Fifty-nine cases involved at least one arrival flight and 17 cases involved at least one departure flight. Also, there were 15 cases that involved violations of wake separation, and 13 cases resulted in the execution of a missed approach. Note that these were losses of separation rather than near misses. At least two cases trigged a TCAS resolution advisory.

Table 3. Characteristics of 70 operational errors

\begin{tabular}{cl}
\hline \hline No. of cases & \multicolumn{1}{c}{ Characteristics } \\
\hline 44 & One or both making final approaches \\
15 & To or from different airports \\
4 & Same airport arrival versus departure \\
59 & One or both arrival \\
17 & One or both departure \\
15 & Wake turbulence \\
2 & TCAS resolution advisory executed \\
13 & Missed approach resulted \\
\hline \hline
\end{tabular}


cases. Fig. 7 shows several minutes of ground track data for two aircraft (AC1 and AC2) leading up to the LOS. Aircraft AC1, represented by the solid line, was an MD82 on a downwind leg. Aircraft AC2, represented by the dashed line, was an MD82 on a base leg. The circles are $3 \mathrm{nmi}$ in diameter at the point of the first LOS. The asterisks are minute markers going back to three minutes before the first LOS. The squares and the grey lines of similar types connecting the squares represent the nominal interior route for each aircraft. Both aircraft are on extended base legs. The sharp turn to the right of AC2 results from a controller intervention.

Fig. 8 shows several minutes of the altitude profiles leading up to the LOS. The zero reference time is at the first LOS, which corresponds to the circles on the ground-track plot. The altitudes are measured relative to MSL. AC1 was descending to $7000 \mathrm{ft}$. AC2 was descending to $5000 \mathrm{ft}$ but not as fast as the controller anticipated, causing the loss of separation. The descent of AC1 and the climb of AC2 a few seconds before the first LOS were responses to controller intervention.

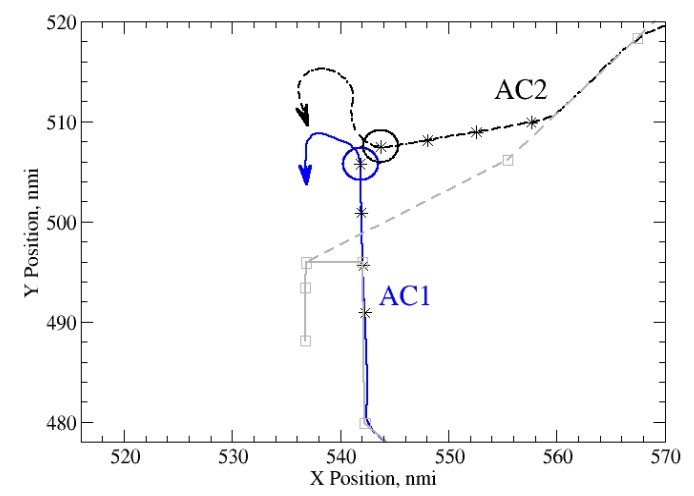

Figure 7. Ground tracks for a sample operational error.

Fig. 9 shows a plot of the horizontal and vertical separations as the two flights lose separation. The origin of this plot represents the point of collision, and the lower left quadrant represents the region of insufficient separation. The discrete points represent the discrete radar samples at intervals of 4.8 seconds. The arrow indicates the evolving direction of the separation with time. The asterisk corresponds to the first LOS.

Fig. 10 shows the alerts generated from the dual conflict prediction algorithm. The time origin is the first-LOS reference time. Each alert marked with an " $x$ " corresponds to a predicted conflict at a radar update. Alert types 1-4 represent predictions using various combinations of FI and DR trajectories. In the case of the FI algorithm, there are only alert types of 0 and 1 while in the DR algorithm there are only alert types of 0 and 4 . Alert type 0 represents the detection of the actual losses of separation. In the case of dual-trajectory algorithm, the alert lead time is the largest alert lead times from alert types 1 to 4 . In the example of Fig. 10, the alert lead time is approximately 95 seconds, and the first predictions of LOS for the four non-zero alert types were atypically at the same time.

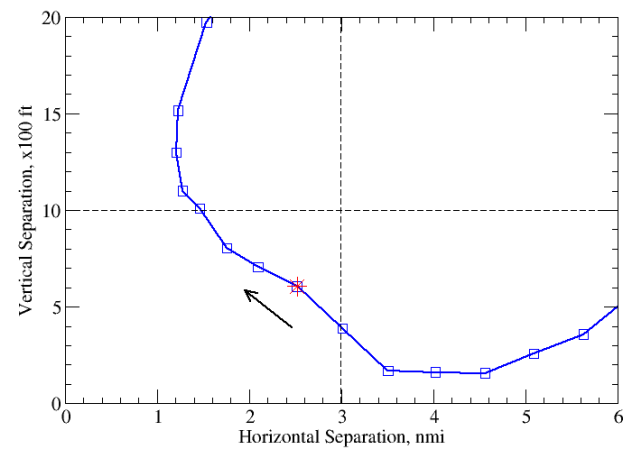

Figure 9. Two-dimensional separation for a sample operational error.

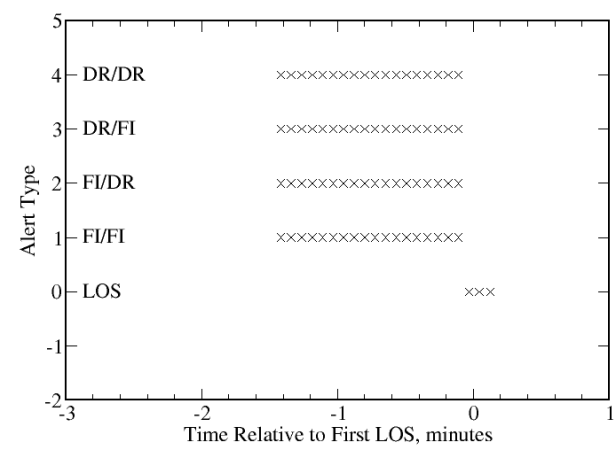

Figure 10. Conflict detection results for a sample operational error.

9

American Institute of Aeronautics and Astronautics 


\section{Alert Cumulative Distribution Function}

The alert lead time for each of the 70 operational error cases was determined for each of the DR, FI, and dual conflict prediction algorithms. The resulting alert cumulative distribution function (CDF) is shown in Fig. 11. The alert CDF provides the cumulative probability of an alert as a function of time relative to the first LOS. The alert lead time is simply the negative of the time relative to the first LOS. Thus, for the FI algorithm, $67 \%$ of the losses of separation were predicted more than 30 seconds in advance. Note that all three algorithms predicted $100 \%$ of the losses of separation by the time of first LOS. The average alert lead times for the DR, FI, and dual algorithms are, respectively, 38, 38, and 44 seconds before first LOS. These alert lead times should be compared with the fact that, among 66 out of the 70 cases, controllers either did not take any action or they acted after a loss of separation had already occurred. The alert CDF, as well as the

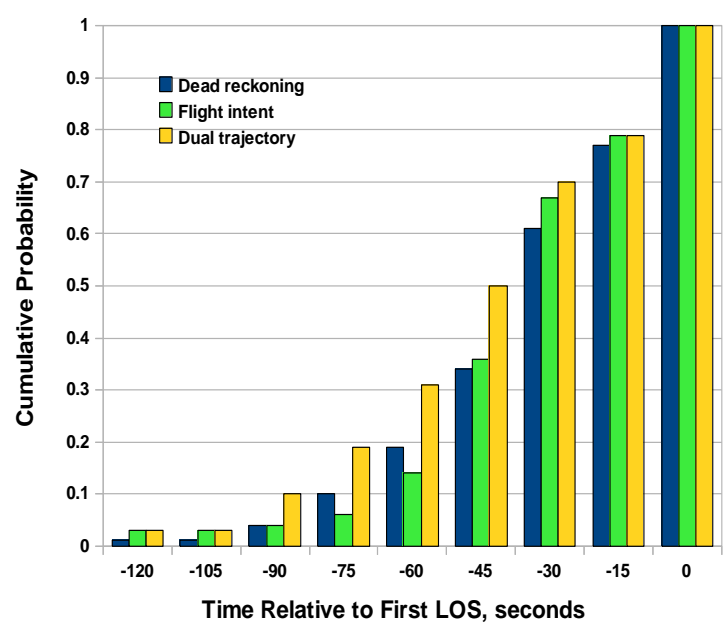

Figure 11. Cumulative distribution function of alert time. average alert lead times, shows that there is no significant improvement of the FI algorithm over the DR algorithm in terms of alert lead time. However, there is a higher probability of detecting a loss of separation sooner for the dual algorithm. This is because the dual algorithm allows for a larger uncertainty in the probing trajectory of an aircraft. However, this more conservative approach will be shown in Sec. III.C to have a much larger false-alert rate.

4. Operational Errors with Small Alert Lead Times

For many operational errors, the alert lead times were small or even zero. In 15 of the 70 cases, all of the three algorithms predicted a loss of separation less than 15 seconds before the first actual loss of separation. Examination of these late-detection cases revealed the characteristics of the encounters that prevented the algorithms from detecting the LOS sooner. Among 8 of the 15 cases, the aircraft made an abrupt descent or climb maneuver due to a controller error that was not expected by the algorithms and was too quick for them to respond. Fig. 12 shows the ground tracks for an example of such late-detection encounters. Fig. 13 shows the altitude profiles for the same encounter with the altitudes again measured above MSL. Aircraft AC1 was a departure that had leveled off at 4000 $\mathrm{ft}$. Aircraft AC2 was an arrival that had leveled off at a cleared altitude of $5000 \mathrm{ft}$. Thus, while the two aircraft converged toward each other horizontally, they maintained the required standard separation of $1000 \mathrm{ft}$ vertically. Following instructions from a controller, the pilot of $\mathrm{AC} 2$ started to descend it from $5000 \mathrm{ft}$ while it was still converging with $\mathrm{AC} 1$ with their horizontal separation close to $3 \mathrm{nmi}$. An alert was generated by the FI algorithm about 14 seconds before the first LOS. Note that if a Terminal TSAFE system was available that allowed the controller to enter the descent command into the system before informing the pilot to descend via voice, an alert could have prevented the controller from descending AC2 until it had passed AC1, and the LOS would have been avoided. This illustrates that it is not possible to provide adequate look-ahead time in all cases without additional intent information.

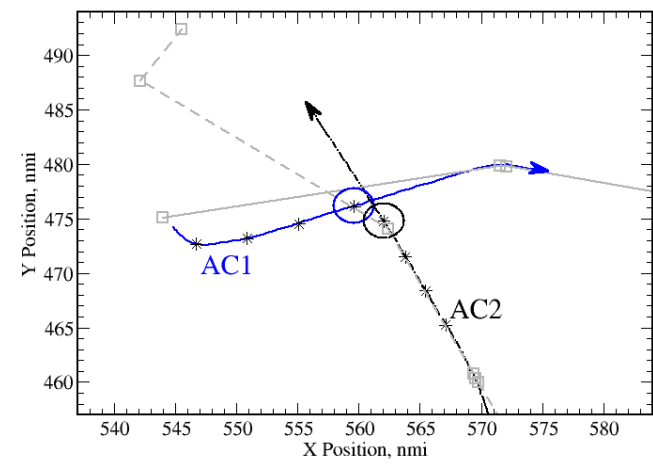

Figure 12. Ground tracks for a sample latedetection operational error.

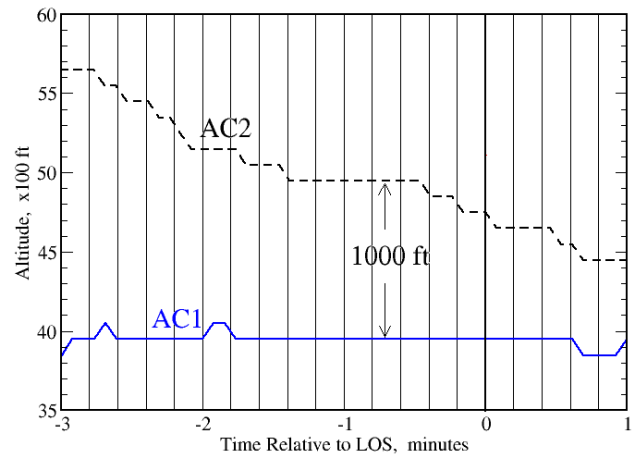

Figure 13. Altitude profiles for a sample latedetection operational error.
10

American Institute of Aeronautics and Astronautics 
The other seven cases with alert lead time less than 15 seconds are classified as follows. There were two cases in which a loss of separation was detected soon after the aircraft appeared in the radar system. There were four cases that involved "blunder" behavior that ignores the flight-intent route, such as abnormal sharp turns while the aircraft was on its base leg of the nominal interior route or flying away from the RNAV departure route. Finally, there was one case that involved an unexpected level off. Thus, the alert lead times for most of the late detections may be improved with the FI algorithm if notice of a maneuver is provided as soon as possible. Again this might be achieved through an interface for controllers to enter an intended descent or climb, a level off, or a turn.

\section{B. False Alerts}

False alerts distract controllers and are potentially dangerous since controllers must verify whether actual problems exist, diverting their attention from whatever genuine conflicts might be developing elsewhere in the airspace. In addition, when false alerts occur too often, they desensitize controllers to true alerts. However, the measures taken for reducing false alerts often decrease alert lead time or even cause missed alerts. To evaluate the conflict prediction algorithms further, we analyzed the false-alert rate for the algorithms using three sets of 30minute track data from DFW TRACON, each of which contains one documented operational error. When real-world TRACON data are analyzed, the classification of alerts is not straightforward.

\section{Classification of Alerts}

Aircraft pairs for which both flights were military or VFR were excluded in the analysis for simplicity since they may require different separation criteria. Also excluded were aircraft pairs for which either flight was a "pop-up" with unknown departure fix, destination fix, or both. Pop-up flights, examples of which include traffic helicopters and survey flights, require special treatment since the separation rules may be different.

As in Ref. 9, although an aircraft pair can have an alert at each radar track update, successive alerts associated with the same aircraft pair will be counted as one alert. Thus, regardless of how many discrete alerts are actually generated for a pair of aircraft, they count as one alert.

After discarding the aircraft pairs mentioned above, the remaining pairs for which alerts were generated can be classified into the usual true and false categories. In the absence of controller action, a true alert is followed by an actual LOS, while a false alert is not. However, when real-world data are used to analyze alerts, as is the case here, this becomes obscured since a controller or a pilot may intervene before a loss of separation occurs. To properly classify alerts into the usual true and false alert categories, we first classify the alerts objectively into LOS and nonLOS alerts. An LOS alert is one that is followed by an actual loss of separation, and a non-LOS alert is not. The non-LOS alerts are then classified subjectively as either valid or invalid alerts as described later in this section. The invalid non-LOS alerts are further divided into leveloff related and non-leveloff related alerts. As seen later, the number of leveloff related invalid alerts are large and may be disturbing to the controller and thus, they are singled out. With this classification, true alerts then consist of LOS and valid non-LOS alerts, while false alerts consist of invalid non-LOS alerts, which include both the leveloff-related and non-leveloff-related alerts. Fig. 14 summarizes the alert classification scheme.

The classification of alerts based on real-world data is difficult due to the nature of terminal operations (vectoring for separation). While aircraft should not be close together in an en route operation, they are purposely placed near the required separation, more so near their final approaches. Thus, the distinction between valid and invalid alerts becomes difficult since alerts beneficial to the controller can become nuisances when they occur too often. An example is a non-LOS alert generated before a leveloff when a loss of separation is predicted because the trajectory used for the prediction does not stop at the cleared altitude. While in many cases the leveloff might be anticipated by the controller, this could also be an unexpected maneuver to avoid a potential conflict. The alert may always be beneficial to the controller whichever scenario it was. However, since the unexpected scenario is not

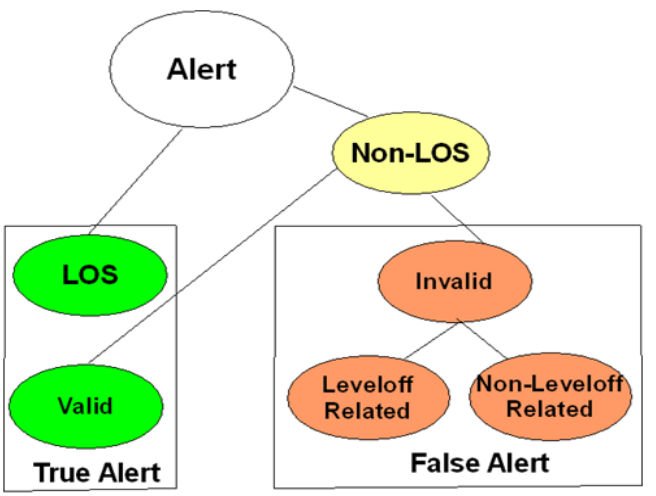

Figure 14. Alert classification. frequent and those leveloff-related alerts occur often, as is seen in the next section, they are put in the invalid category. Note that the classification has no effect on a real TSAFE system in the sense that if the cleared altitude is a part of the available intent, (e.g. when it is entered through an optional interface), the alert will not be present. Otherwise, it would be there to help the controller. More details on the non-LOS alerts are described next. 


\section{Valid non-LOS alerts}

Valid non-LOS alerts are those that are not subsequently followed by actual losses of separation because controllers or pilots intervene with maneuvers. The maneuvers can be vertical maneuvers involving a climb or descent with significant change in the vertical rate, horizontal maneuvers involving a non-procedural turn, or speed maneuvers involving a sharp change of speed. The non-procedural turns are those that do not correspond to the standard operational procedures. Standard turns include the downwind-to-base, base-to-final, or the RNAV departure turns.

An example of a valid non-LOS alert is shown by the altitude profiles in Fig. 15, where the altitudes of two departure aircraft $\mathrm{AC} 1$ and $\mathrm{AC} 2$ are plotted. The solid and dashed lines represent the actual altitude profiles of $\mathrm{AC} 1$ and $\mathrm{AC} 2$, respectively. The thick lines of similar types are the predicted

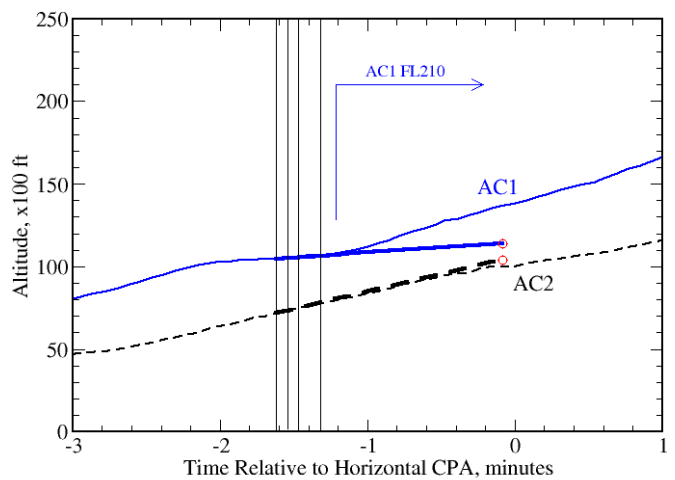

Figure 15. Altitude profiles for a pair of aircraft with a valid non-LOS alert.

altitudes of the two aircraft and the small circles indicate the first predicted LOS. The arrow indicates that AC1 was assigned a cleared altitude at FL210 (flight level 21000 pressure altitude) by a controller. The vertical thin lines indicate the times at which the conflicts were predicted. During these predictions, AC1 was leveled at $10000 \mathrm{ft}, \mathrm{AC} 2$ was climbing, and horizontally they were approaching $3 \mathrm{nmi}$ separation (tracks are not shown). Their headings were at an angle of about $33^{\circ}$ and they followed their flight plan routes closely. The time of the first prediction was about 1.6 minutes away from the horizontal Closest Point of Approach (CPA) and the time to the predicted LOS was about 1.5 minutes as indicated in Fig. 15. While the two aircraft got within $3 \mathrm{nmi}$ horizontally, they maintained more than $1000 \mathrm{ft}$ separation vertically. An actual loss of separation was avoided due to the change in climb rate from about $600 \mathrm{fpm}$ to $2700 \mathrm{fpm}$ as AC1 was issued the clearance to climb to FL210 after the prediction of the potential LOS. This is deemed a valid alert since the controller intervened after the prediction of LOS.

\section{Leveloff-related alerts}

Invalid leveloff-related non-LOS alerts are those that are not subsequently followed by actual losses of separation because the trajectories used to predict the conflicts are inaccurate for lack of flight intent data about the cleared altitudes. Three types of alerts in this category are possible:

1) Alerts generated because the predicted trajectories do not level off at the cleared altitudes.

2) Alerts generated while one of the aircraft is just leveling off but its vertical rate is not zero yet as a result of the lagging due to data smoothing.

3) Alerts generated for aircraft that are supposedly in level flight, but outlying altitude points cannot be rounded to the cleared altitudes because altitude rounding cannot be applied without knowing the cleared altitudes as parts of the flight intent data.

As will be seen in Sec. III.B.3 below, if these alerts are not properly isolated and avoided, their quantity can be large enough to become overwhelming to controllers.

Many altitude clearances in terminal airspace are anticipated from the approach procedures or standard controller techniques. For example, it is typical that an aircraft needs to level off at $4000 \mathrm{ft}$ or $5000 \mathrm{ft}$ before turning onto the final approach courses. Thus, altitude clearances can be available to TSAFE ahead of time. However, this is not the case today since altitude clearances are only communicated via voice in the TRACON. In this research, inferred altitude clearances as discussed in Sec. II.C.3 are used to study the importance of including such flight-intent information. In the Next Generation Air Transportation System, where altitude clearances will likely be explicitly entered by the controller, TSAFE may avoid many invalid leveloff-related alerts. In the near term, a controller interface could be added to enter altitude clearances optionally when

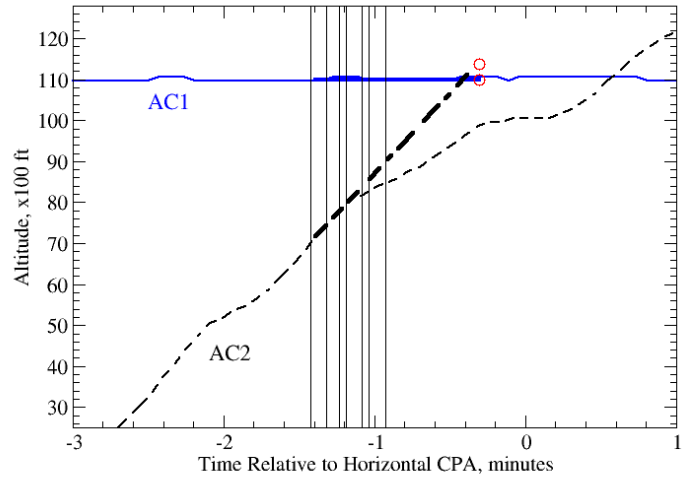

Figure 16. Altitude profiles for a pair of aircraft with an invalid leveloff-related alert. workload permits. 
An example of invalid leveloff-related non-LOS alerts is shown in Figs. 16, where the altitudes above MSL of aircraft $\mathrm{AC} 1$ and $\mathrm{AC} 2$ are plotted against the time relative to the horizontal closest appoint of approach. As in Fig. 15 , the solid and dashed lines are for AC1 and AC2 respectively and the thick lines of similar types are the predicted altitude profiles. The vertical thin lines indicate consecutive predictions of potential conflicts. AC1 was level at $11000 \mathrm{ft}$ while AC2 was climbing and intended to level at a cleared altitude of $10000 \mathrm{ft}$ (not entered into the system). Horizontally both aircraft followed their flight plan route closely with their tracks being about to cross each other. Thus, a horizontal separation less than $3 \mathrm{nmi}$ was unavoidable. However, for lack of the flight intent of the altitude clearance at $10000 \mathrm{ft}, \mathrm{AC} 2$ was predicted to climb through that altitude, yielding a predicted conflict as indicated by the small circles in Fig. 16.

\section{Non-leveloff-related alerts}

Invalid non-leveloff-related non-LOS alerts are technically invalid alerts that are not leveloff related. They are not subsequently followed by actual losses of separation because the prediction trajectories fail to model the standard operation procedures or there are still unfiltered outlying track data.

An example of an invalid non-leveloff-related alert is shown in Fig. 17, where the tracks of aircraft AC1 and $\mathrm{AC} 2$ are plotted. The solid and dashed lines correspond to the actual tracks of aircraft $\mathrm{AC} 1$ and AC2. The grey lines of similar types are the flightintent (nominal interior) routes. The dark solid and dashed lines are predicted trajectories using the DR algorithm. The dark circles of $3 \mathrm{nmi}$ in diameter indicate the first prediction of a potential LOS while the thin circles indicate the actual CPA. Because the

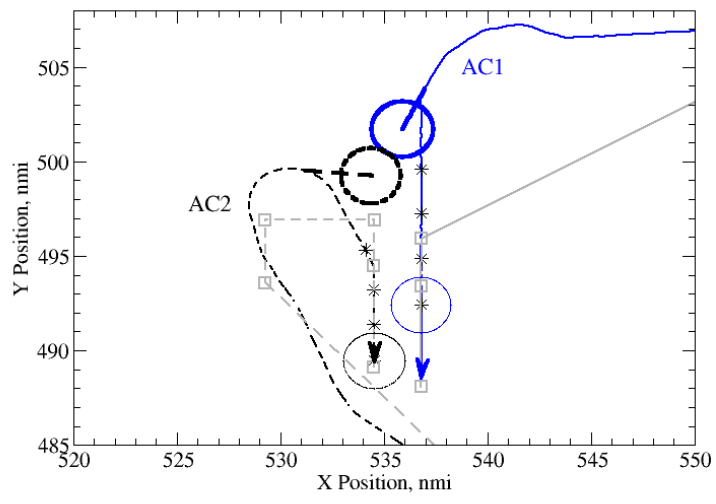
aircraft followed the nominal interior routes instead of

Figure 17. Ground tracks for a pair of aircraft with an invalid non-leveloff-related alert.

the DR trajectories, the predicted LOS never materialized. Note that alerts generated by the DR and dual-trajectory algorithms, designed for comparison with the FI algorithm, were classified by the same scheme.

\section{False Alerts}

Three 30-minute data sets of recent arrival and departure operations at DFW TRACON are used for false-alert analysis. These data sets are summarized in Table 4 with each set containing one documented operational error.

The average aircraft count in the data set is the average number of aircraft in the TRACON at a given time, which measures how busy the TRACON was. The number of LOS alerts for each data set generated based on the separation criteria described in Sec. II.A is also presented. This number is the same for all of the algorithms, in agreement with the earlier result that there are no missed alerts for each algorithm. It is interesting also to note that the higher the average traffic density in the TRACON, the higher the number of LOS alerts.

Excluding the alerts corresponding to the documented operational errors, a total of 21 LOS alerts for the three sets together were found. Table 5 shows the characteristics of these LOS alerts that were not reported as operational errors.
Table 5 Characteristics of LOS alerts not reported as operational errors

\begin{tabular}{cl}
\hline \hline No. of cases & \multicolumn{1}{c}{ Characteristics } \\
\hline 13 & $\begin{array}{l}\text { LOS occurred when one or both of the aircraft on parallel final } \\
\text { approaches were not established on the localizer. }\end{array}$ \\
1 & $\begin{array}{l}\text { LOS occurred because one of the aircraft on parallel final approaches } \\
\text { stepped to a close-by "departure" runway (which is actually legal). }\end{array}$ \\
3 & $\begin{array}{l}\text { LOS occurred due to violation of wake turbulence separation on the } \\
\text { same runway. } \\
\text { Other violations of the separation standards between two arrival flights. }\end{array}$ \\
\hline
\end{tabular}

13

American Institute of Aeronautics and Astronautics 
Most of them involved small violations of the separation standards when one or both aircraft was turning on the final approach. Inspection of the weather conditions and radar tracks suggests that the associated aircraft were likely conducting visual approaches and thus, these LOS alerts were likely not actual operational errors.

Table 6 shows the number of non-LOS alerts per hour of different types generated from the FI, DR, and dualtrajectory detection algorithms with or without the use of inferred altitude clearances (IACs). The types of invalid alerts are LR for leveloff related and NLR for non-leveloff related. The results can be summarized as follows:

Table 6 Number of non-LOS alerts per hour of different types for different algorithms

\begin{tabular}{|c|c|c|c|c|c|c|c|c|c|}
\hline & \multicolumn{3}{|c|}{ FI } & \multicolumn{3}{|c|}{ DR } & \multicolumn{3}{|c|}{ Dual } \\
\hline & \multirow[t]{2}{*}{ Valid } & \multicolumn{2}{|c|}{ Invalid } & \multirow[t]{2}{*}{ Valid } & \multicolumn{2}{|c|}{ Invalid } & \multirow[t]{2}{*}{ Valid } & \multicolumn{2}{|c|}{ Invalid } \\
\hline & & LR & NLR & & LR & NLR & & LR & NLR \\
\hline With IAC & 7.3 & 0 & 2.0 & 5.3 & 0 & 19 & 8.7 & 0 & 29 \\
\hline No IAC & 6.0 & 18 & 4.7 & 4.0 & 19 & 16 & 10 & 25 & 33 \\
\hline
\end{tabular}

1) Apart from the FI algorithm with IAC, the number of valid alerts is small compared to the number of false (invalid) alerts.

2) Use of the IACs removes the leveloff-related false alerts.

3) The number of false (invalid) alerts for the FI algorithm is significantly less.

The difference in false-alert rates for different algorithms can also be seen in the chart shown in Fig. 18. The effect of using inferred altitude clearances is also shown. Compared with the dual algorithm, the false-alert rates for the DR and FI algorithms reduce by $40 \%$ and $61 \%$, respectively, without the use of the IAC in the algorithms. The corresponding reductions become $34 \%$ and $93 \%$, respectively, with the use of the IAC in the algorithms.

The total number of false alerts for the FI algorithm with inferred altitude clearances for the three data sets is three. One of them resulted from some outlying data points that were not filtered out and involved a VFR flight. The other two appeared to result from inadequate modeling of the standard operational procedures.

The significant reduction of false alerts from the dual to the FI algorithm and as a result of including IAC indicates that the use of nominal interior routes, RNAV departure routes, and inferred altitude clearances remove a majority of the false alerts. The DR algorithm generates considerably more false alerts than the FI algorithm because it does not take into account the standard operational procedures. The dual algorithm generates significantly more false alerts as compared to the DR

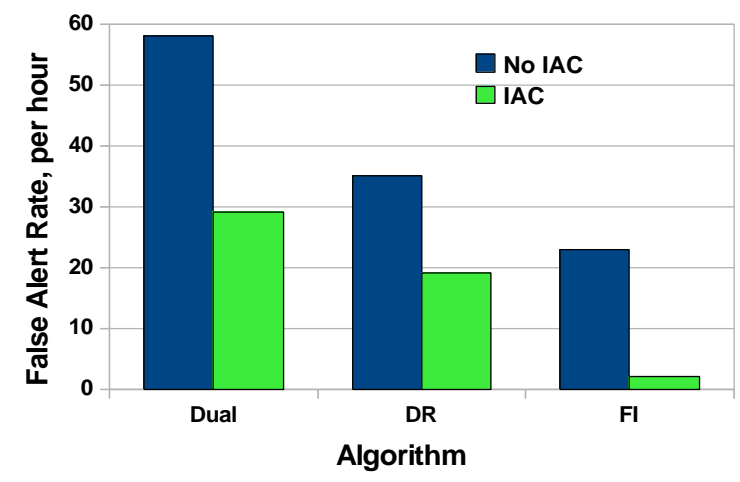

Figure 18. False-alert rates for different detection algorithms.

algorithm because it includes false alerts generated by both DR and FI trajectory algorithms. The altitude envelopes used in the dual algorithm also contribute to the higher false alert rate. The leveloff-related false alerts are completely removed by introducing the inferred altitude clearances. The removal of the false alerts reduces the falsealert rate to a manageable level. These results show clearly the importance of including some additional intent information for short term conflict prediction in the TRACON.

\section{Conclusions}

A new tactical conflict detection flight-intent algorithm for terminal airspace has been studied. The FI algorithm uses a single analytic trajectory that is based on available flight intent and current state information of the aircraft with alerts determined by the standard separation criteria of the FAA in terminal airspace. The flight intent information includes the flight plan, part of which is the RNAV departure route, the nominal interior route, the inferred altitude clearances, and some ground-speed bounds. The FI algorithm is compared with a dual algorithm that imitates the en-route TSAFE algorithm and a DR (dead-reckoning) algorithm that imitates Conflict Alert. The comparison is done through analysis of the false-alert rates and alert lead times from fast-time simulation of track data of three sets of departure and arrival operations and 70 operational error cases from DFW TRACON during the period between January 2007 and April 2009. The FI algorithm shows significantly reduced false alerts without much degradation of the alert lead time. The false-alert rate for the FI algorithm was reduced to two alerts per hour, 
and the alert lead time average over 70 actual TRACON operational errors was 38 seconds. Smoother track data and better flight intent data available in the future would only improve the results.

While the false-alert rate may be at a manageable level, more extensive tests with broader data from other facilities, as well as human-in-the-loop simulation experiments, are still needed. It is also good to have a direct comparison between the results of terminal TSAFE and Conflict Alert. Unfortunately such comparison is not straightforward since, among other things, the separation criteria are quite different, and Conflict Alert is often inhibited in areas of frequent false alerts. Work in these areas is in progress. It appears that altitude clearances play an important role as additional flight intent information for terminal TSAFE. Further research on how to obtain this information efficiently is needed.

\section{References}

${ }^{1}$ JPDO, "Concept of Operations for the Next Generation Air Transportation System", version 2.0, June 2007.

${ }^{2}$ Erzberger, H., "The Automated Airspace Concept", Proceedings of the fourth USA/Europe Air Traffic Management R\&D Seminar, Santa Fe, New Mexico, USA, December 3-7, 2001.

${ }^{3}$ Erzberger, H., and Paielli, R.A., "Concept for Next Generation Air Traffic Control System”, Air Traffic Control Quarterly, vol. 10, no. 4, 2002, pp.355-378.

${ }^{4}$ Erzberger, H., "Transforming the NAS: The Next Generation Air Traffic Control System", NASA/TP-2004-212828, October 2004.

5“Introduction to TCAS II Version 7", Federal Aviation Administration, Washington D.C., Nov. 2000.

${ }^{6}$ Erzberger, H., Davis, T.J., and Green, S.M., "Design of Center-TRACON Automation System", AGARD Meeting on Machine Intelligence in Air Traffic Management, Research and Technology Organization, May 1993.

${ }^{7}$ Paielli, R.A. and Erzberger, H., "Tactical Conflict Detection Methods for Reducing Operational Errors", Air Traffic Control Quarterly, Vol. 13(1), 2005, pp 83-106.

${ }^{8}$ Paielli, R.A. and Erzberger, H., "Analysis of False-Alert Rates for a Tactical Conflict Detection System”, AIAA Paper ATIO-05, Sept. 2005.

${ }^{9}$ Paielli, R.A., Erzberger, H., Chiu, and Heere, K.R., "Tactical Conflict Alerting Aid for Air Traffic Controllers", AIAA, Journal of Guidance, Control, and Dynamics, Vol.32, No.1, 2009, pp 184-193.

${ }^{10}$ Erzberger, H. and Heere, K., "Algorithm and Operational Concept for Resolving Short Range Conflicts", University of California, Santa Cruz. Paper No. 383, ICAS-08, Sept. 2008.

${ }^{11}$ Paielli, R.A., "Tactical Conflict Resolution using Vertical Maneuvers in Enroute Airspace", AIAA, Journal of Aircraft, Vol.45, No.6, 2008, pp 2111-2119.

${ }^{12}$ Tang, H., Denery, D., Erzberger, H., and Paielli, R.A., "Tactical Separation Algorithms and Their Interaction with Collision Avoidance Systems", AIAA, GNC conference, AIAA-2008-6973, August, 2008.

13 "EUROCONTROL Specification for Short Term Conflict Alert", Edition No. 1.0, Eurocontrol Experimental Centre, 22 November 2007.

${ }^{14}$ "National Airspace System En Route Configuration Management Document, Host-A5f1.3: Computer Program Functional Specifications, Automatic Tracking", Federal Aviation Administration, NAS-MD-321 2002.

${ }^{15}$ Nolan, M. S., Fundamentals of Air Traffic Control, 4th ed., Brooks-Cole, Belmont, California, 2004, pp. $342,382$.

${ }^{16}$ Air Traffic Control FAA Order JO 7110.65S, Federal Aviation Administration, Washington, DC. Feb. 2008. (Published online at http://www.faa.gov).

${ }^{17}$ Aeronautical Information Manual, Federal Aviation Administration, Washington, DC. Feb. 2010. (Published online at http://www.faa.gov) .

${ }^{18}$ Swenson, H. N., Hoang, T., Engelland, S., Vincent, D., Sanders, T., Sanford, B., and Heere, K., "Design and Operational Evaluation of the Traffic Management Advisor at the Fort Worth Air Route Traffic Control Center," 1st USA/Europe Air Traffic Management R\&D Seminar, Saclay, France, June 1997.

19 "User Manual for the Base of Aircraft Data (BADA)", Revision 3.6, EEC Note No. 10/04, ACE-C-E2, Eurocontrol Experimental Centre, 4 July 2004.

${ }^{20}$ Lindsay, K. S., "Currency of Flight Intent Information and Impact on Trajectory Accuracy", FAA/Eurocontrol Technical Interchange Meeting on Shared Flight Intent Information and Aircraft Intent Data, Federal Aviation Administration, Washington, DC. Oct. 2000. 